\title{
Noninvasive Blood Glucose Measurement Based on NIR Spectrums and Double ANN Analysis
}

\author{
D. X. Guo, Y. Z. Shang, R. Peng, S. S. Yong, X. A. Wang* \\ The Key Laboratory of Integrated Microsystems, Peking University Shenzhen Graduate School, Shenzhen, China \\ Email: 454071436@qq.com
}

Received May 2015

\begin{abstract}
This paper presents a new noninvasive blood glucose monitoring method based on four near infrared spectrums and double artificial neural network analysis. We choose four near infrared wavelengths, $820 \mathrm{~nm}, 875 \mathrm{~nm}, 945 \mathrm{~nm}, 1050 \mathrm{~nm}$, as transmission spectrums, and capture four fingers transmission PPG signals simultaneously. The wavelet transform algorithm is used to remove baseline drift, smooth signals and extract eight eigenvalues of each PPG signal. The eigenvalues are the input parameters of double artificial neural network analysis model. Double artificial neural network regression combines the classification recognition algorithm with prediction algorithm to improve the accuracy of measurement. Experiments show that the root mean square error of the prediction is between $0.97 \mathrm{mg} / \mathrm{dL}-6.69 \mathrm{mg} / \mathrm{dL}$, the average of root mean square error is $3.80 \mathrm{mg} / \mathrm{dL}$.
\end{abstract}

\section{Keywords}

Noninvasive, Blood Glucose, NIR, ANN

\section{Introduction}

Diabetes has become a common disease in modern society. The blood glucose is too high or too low both will cause a significant impact on health, and the complications of diabetes are very serious, such as liver cirrhosis and neuropathy (Garcia-Compean et al. 2009, Mitrović et al. 2014) [1] [2]. Diabetes is a disease difficult to cure, only through diet or insulin injections to control blood glucose. It is necessary to know their blood glucose realtime and accurately for the patients. However, the current method to measure blood glucose is mainly direct drawing blood from patients, which is a electrochemical way. Using invasive way to measure blood glucose for a long-term, the patients suffer from great physical pains, and the risk of infection increases. All kinds of testing strips of invasive blood glucose are expensive disposable consumables. These factors are not conducive to patients to facilitate and timely understand their blood glucose condition (Ramachandran et al. 2012) [3].

In order to overcome the disadvantages of invasive blood glucose, the non-invasive blood glucose measure-

\footnotetext{
"Corresponding author.
}

How to cite this paper: Guo, D.X., Shang, Y.Z., Peng, R., Yong, S.S. and Wang, X.A. (2015) Noninvasive Blood Glucose Measurement Based on NIR Spectrums and Double ANN Analysis. Journal of Biosciences and Medicines, 3, 42-48. 
ment has become a hot academic research topic (Ferrante do Amaral and Wolf 2008, Vashist 2012) [4] [5]. The researches of using near infrared spectroscopy for noninvasive blood glucose measurement are also increasing recently (Unnikrishna Menon et al. 2013, Ramasahayam et al. 2013) [3] [6]. But on the choice of near-infrared wavelengths, these studies generally use single wavelength or similar wavelengths. For instance, the NIR lights with high absorptivity are always taken as transmission spectrums or reflectance spectrums (Lam et al. 2010, Maruo et al. 2003, Malin et al. 1999) [7]-[9]. This study proposes using four-channel $820 \mathrm{~nm}, 875 \mathrm{~nm}, 945 \mathrm{~nm}$ and $1050 \mathrm{~nm}$ infrared light as the transmission wavelengths. After extracting the eigenvalues of each PPG signal with the wavelet transform algorithm, the estimation model is developed with double artificial neural network.

\section{Principle Analysis}

\subsection{PPG Signals}

Though analyzing the fingertip photoplethysmography (PPG) signals, the physiological information of human blood and tissues can be extracted (Elgendi 2012, Ubeyli et al. 2007) [10] [11]. When a bunch of near-infrared light transmit through the human fingertip and reach the corresponding photoelectric sensor, the absorption of skin tissue and blood will cause the beam weaken, thus the photoelectric signal of photoelectric sensors will be a certain of attenuated.

The near-infrared light absorption of skin tissue, muscle, bone, and venous blood of human fingertip in a short time remains constant. Only the changes of arterial blood will cause beam attenuation changes. As the heart pumps blood, blood will periodically circulation, the human fingertip artery blood volume will follow cyclical changes. When the body is systolic, the heart blood is supplied to the whole body, and the finger arterial blood will become filled. At this time, the absorption of the near-infrared light is strongest and electrical signals of photoelectric sensors are weakest. On the contrary, the absorption of the near-infrared light is strongest. In this way, the system can get cyclical PPG signals.

As shown in Figure 1, the PPG signal of fingertip consists of two parts: (1) DC component: It reflects the near-infrared light absorption of fingertip skin, muscle, bone and venous blood. The DC component essentially remains unchanged; (2) AC component: It reflects the near-infrared light absorption of fingertip arterial blood. The AC component will change periodically with heart rate.

Not only do the fingertip PPG signals include the blood glucose information, but also include the information of other tissues. In order to extract the effectively spectral information, we need to remove the noise caused by other tissues such as fingertip skin tissue and venous blood.

According to Lambert Beer's law, set $O D_{\lambda}$ as the optical absorbance at time $t_{i}, O D_{\lambda}^{\prime}$ is the optical absorbance at time $t_{i+1}$, then

$$
\begin{aligned}
& O D_{\lambda}=\log \left(I_{0} / I_{\lambda}\left(t_{i}\right)\right) \\
& O D_{\lambda}^{\prime}=\log \left(I_{0} / I_{\lambda}\left(t_{i+1}\right)\right)
\end{aligned}
$$

where $I_{\lambda}$ is the transmitted intensity of light at time $t_{i} ; I_{\lambda+1}$ is the transmitted intensity of light at time $t_{i+1}$;

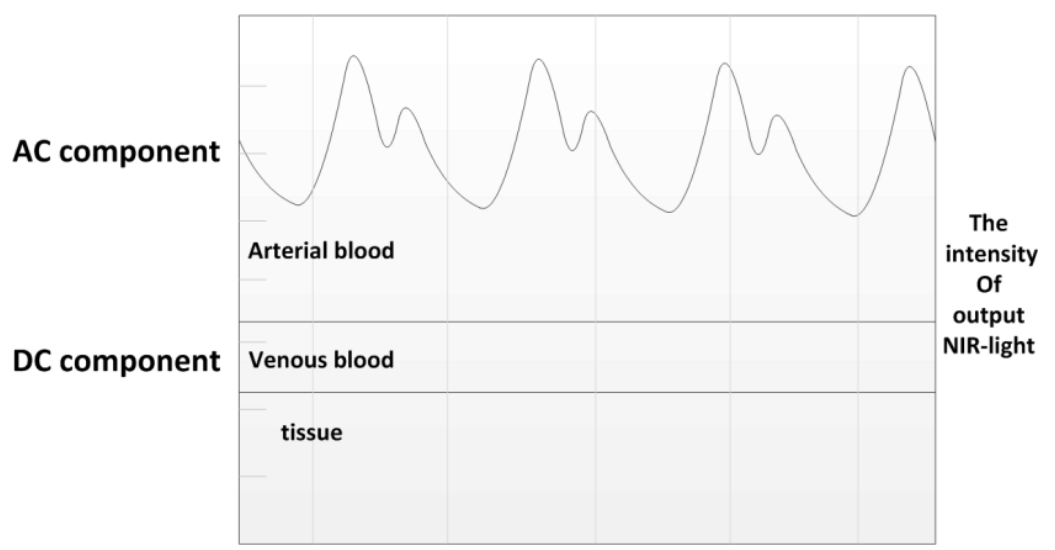

Figure 1. The PPG signal of fingertip. 
the difference of $t_{i}$ and $t_{i+1}$ is $\Delta t$; the difference between $O D_{\lambda}$ and $O D_{\lambda}^{\prime}$ is $\Delta O D_{\lambda}$ (Du et al. 2004) [12], which eliminates the background noise:

$$
\Delta O D_{\lambda}=O D_{\lambda}-O D_{\lambda}^{\prime}=\log \frac{I_{\lambda}\left(t_{i}\right)}{I\left(t_{i+1}\right)}=\log \left[1+\frac{\Delta I_{\lambda}\left(t_{i}\right)}{I_{\lambda}\left(t_{i+1}\right)}\right]
$$

\subsection{The Selection of Wavelengths}

The molecular formula of glucose contains a multiple of O-H, C-H chemical bond. In the wave bands of $935 \mathrm{~nm}$ - $960 \mathrm{~nm}$ and $1040 \mathrm{~nm}-1080 \mathrm{~nm}$ exist the near infrared spectrums peak absorption of glucose (Ramasahayam et al. 2013) [13].

The wavelengths of $945 \mathrm{~nm}$ and $1050 \mathrm{~nm}$ are selected as key wavelengths, which are the peak wavelengths. It can reflect near-infrared light absorption of blood glucose.

Other selected wavelengths are $820 \mathrm{~nm}$ and $875 \mathrm{~nm}$, whose absorption is relatively low. These wavelengths are taken as reference wavelengths. PPG signals caused by key wavelengths not only contain the information of blood glucose, but also other substances information. Combining key reference wavelength with wavelength to develop model can effectively eliminate the influence of other substances.

According to Lambert Beer's law

$$
A=k^{*} c^{*} d
$$

In which, $\boldsymbol{k}$ is the molar absorption coefficient, $\boldsymbol{d}$ is optical path that means the thickness of the fingertip, $\boldsymbol{c}$ is liquid concentration that means blood glucose concentration here. For the four PPG signals of one person at the same moment, the blood glucose concentration $\boldsymbol{c}$ and the thickness of the fingertip $\boldsymbol{d}$ is the same.

\section{The System Design}

\subsection{The System of Gathering PPG Signals}

The hardware of near-infrared non-invasive blood glucose estimated system mainly includes three parts. (1) The module to gather PPG signals. The LED near infrared sensors are used to complete the gathering of PPG signals; (2) The module to preprocess PPG signals, which includes the analog filter circuits, signal amplifying circuits, analog-to-digital conversion circuits and MCU. (3) The module to build the estimation model. The wavelet transform and artificial neural network are applied to develop the model. The block diagram of the noninvasive blood glucose measurement system is shown in Figure 2, The PPG signals after removing baseline drift and smoothing is shown in Figure 3.

In the module to gather PPG signals, fingertips are placed on the clips. Near infrared LEDs emitted sensor is located in the upper of clip, which can emit corresponding wavelengths of near-infrared light. After through the finger, the light will reach corresponding photoelectric sensor located in the bottom of clip. With fingertip blood

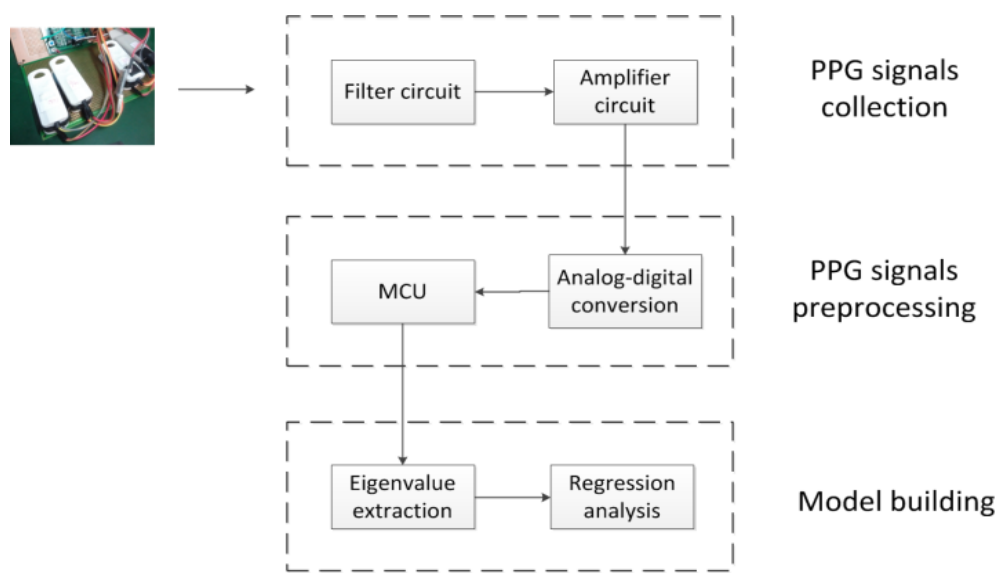

Figure 2. The block diagram of the noninvasive blood glucose measurement system. 


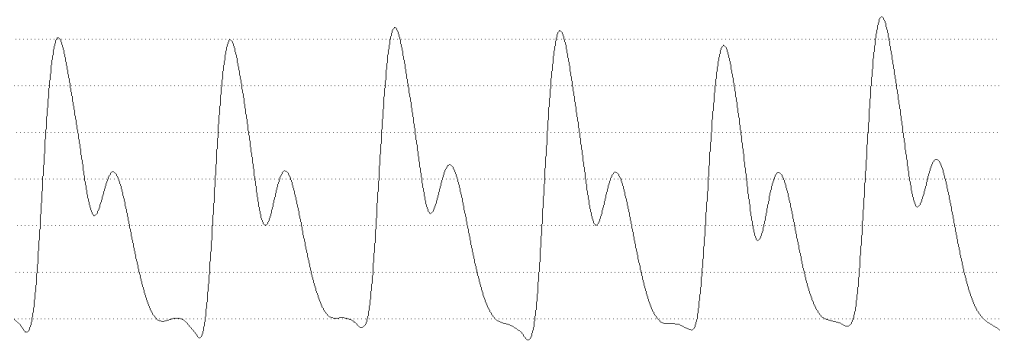

Figure 3. The PPG signals after removing baseline drift and smoothing.

vessels periodic filling and void, the system can get cyclical PPG signals.

\subsection{Double Artificial Neural Network Analysis}

\subsubsection{Analysis Methods of Artificial Neural Networks}

The stoichiometric method commonly used in the near infrared spectral analysis includes linear correlation and non-linear correlation analysis. The linear correlation analysis mainly includes: multiple linear regression analysis (MLR), principal component regression (PCR), partial least squares (PLS), etc. The non-linear correlation analysis mainly includes: artificial neural network (ANN) and support vector machine (SVM) (Lam et al. 2010, Yadav et al. 2014, Barman et al. 2010) [7] [14] [15]. The ANN classification recognition-prediction double algorithms are used to develop the estimation model.

As shown in Figure 4, typical PPG signals should have the main wave P, the dicrotic wave T, the main trough A and the sub-trough V. The wavelet transform algorithm is used to remove the baseline drift of PPG signals and smooth it.

\subsubsection{Double ANN Analysis}

To improve the accuracy of the regression analysis, the study uses double ANN analysis: one is based on artificial neural network classification algorithm, and the other is based on artificial neural network prediction algorithm (Ping et al. 2005) [16].

First, the test data are applied to the network trained by classification algorithm, which will get the result R1. The result R1 is used to estimate the range which the blood glucose concentration value belongs.

Then, the test data are applied to the network trained by prediction algorithm, which will get the result R2.

If R2 belongs the interval judged by R1, the R2 is right and retained as the predicted results. On the contrary, the R2 is considered inaccurate and discarded.

Since every time will gather enough PPG signals data that obtain a plurality of predicted results. After removing the maximum and minimum values of the results, the remaining data are averaged as the final predicted result.

\subsection{The Experiment Conclusion}

5 glucose tolerance test normal and healthy men participate in the experiment, their age distribute in 23 to 26 . In the three and a half hours from the morning. Every 20 minutes, the subjects are measured twice using the PPG signals gathering system, and it will obtain multiple sets of PPG signals data. The first set of data are recorded as $A_{i}$ and used to train the artificial neural network; the second set of data are recorded as $B_{i}$ and used to verify the system. It will measure ten times and Roche Excellent type glucose meter is used to measure the real blood glucose level $C$ at the same time, that are recorded as $C_{\text {real-i, }}$, where $i$ is the number of measurement.

It uses $A_{i}$ and real blood glucose concentration $C_{\text {real }-i}$ for training the net. Finally it uses PPG signals that are not involved in the training of the net and the trained net for estimating, and gets the estimated blood glucose $C_{\text {est }-i}$.

$\mathrm{n}_{1}=8, \mathrm{n}_{2}=8, \mathrm{n}_{3}=7, \mathrm{n}_{4}=10, \mathrm{n}_{5}=7$, $\mathrm{n}$ is the number of the estimation model can output the blood glucose estimation.

As is shown in Figure 5, it is the compare of the estimated values and the real values of blood glucose.

To judge the accuracy of the estimation, a root mean square error index is introduced: 


$$
R M S E=\sqrt{\frac{\sum_{i=1}^{n}\left(x_{i}-y_{i}\right)^{2}}{n}}
$$

where $x_{i}=C_{\text {est- }}$, which is the estimated blood glucose, $y_{i}=C_{\text {real-i }}$, which is the real blood glucose. After calculating $\mathrm{RMSE}_{1}=4.10 \mathrm{mg} / \mathrm{dL}, \mathrm{RMSE}_{2}=4.64 \mathrm{mg} / \mathrm{dL}, \mathrm{RMSE}_{3}=2.58 \mathrm{mg} / \mathrm{dL}, \mathrm{RMSE}_{4}=6.69 \mathrm{mg} / \mathrm{dL}, \mathrm{RMSE}_{5}=$ $0.97 \mathrm{mg} / \mathrm{dL}$. The average of root mean square error is $3.80 \mathrm{mg} / \mathrm{dL}$.

To further verify the accuracy of the estimation, Clarke error grid analysis (CEG) is applied. As is shown in Figure 6, the final result is $100 \%$ distributed in the region A, which is considered to be completely accurate in

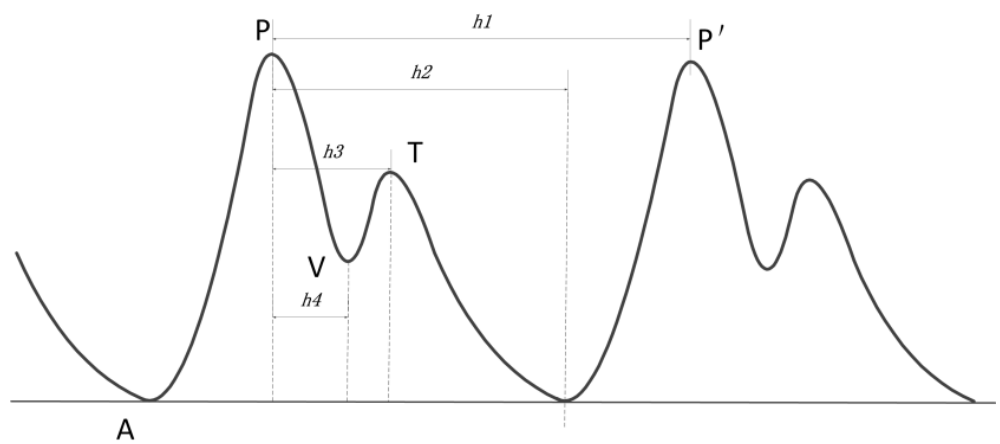

Figure 4. The eigenvalues of PPG signals.

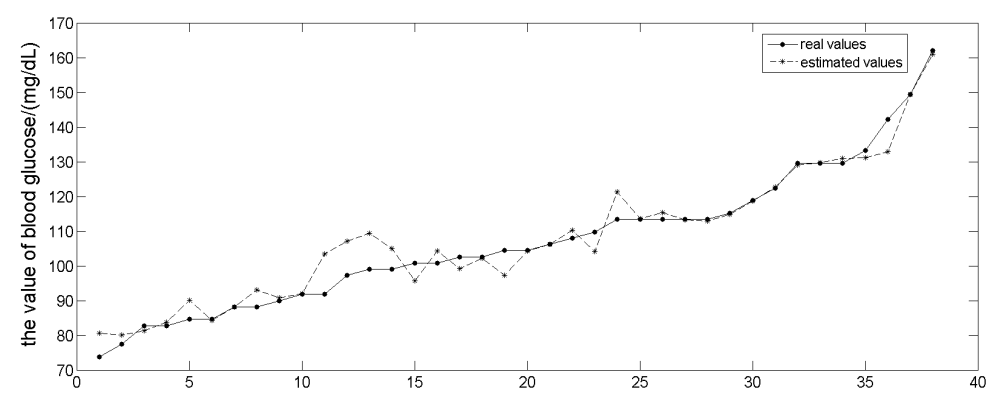

Figure 5. The estimated values compared with the real values of blood glucose.

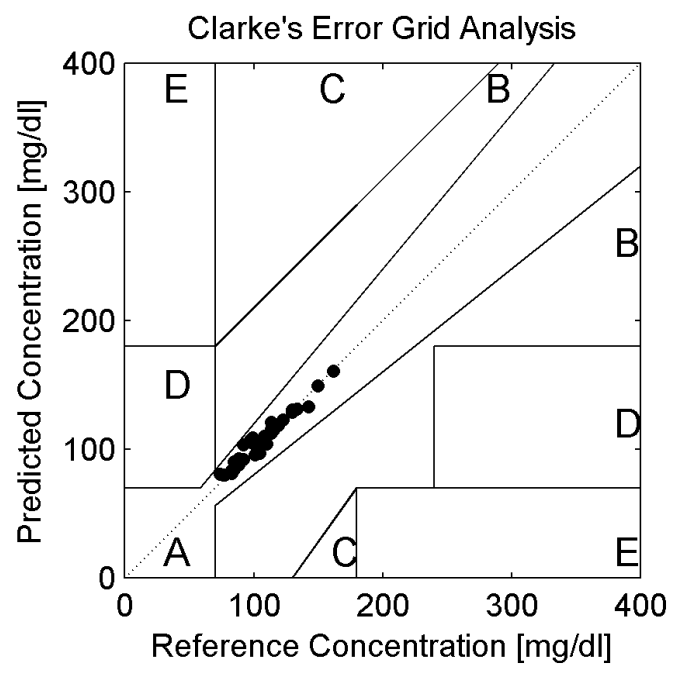

Figure 6. The Clarke error grid analysis of predicted blood glucose and reference blood glucose (mg/dl). 
clinical (Kovatchev et al. 2004) [17].

\section{Conclusions}

We use the wavelengths of $820 \mathrm{~nm}, 875 \mathrm{~nm}, 945 \mathrm{~nm}$ and $1050 \mathrm{~nm}$ as near-infrared light as transmitted wavelengths, gather four PPG signals of fingertips, and design a set of non-invasive blood glucose estimating system according to Lambert Beer's law. And double artificial neural network algorithms are used to build model with personal PPG signals. The average root mean square error is $3.80 \mathrm{mg} / \mathrm{dL}$.

In the future studies, we will increase the number of test subjects, and consider more factors, such as environmental humidity, temperature during the experiment.

\section{References}

[1] Garcia-Compean, D., Jaquez-Quintana, J.O., Gonzalez-Gonzalez, J.A. and Maldonado-Garza, H. (2009) Liver Cirrhosis and Diabetes: Risk Factors, Pathophysiology, Clinical Implications and Management. World Journal of Gastroenterology, 15, 280-288. http://dx.doi.org/10.3748/wjg.15.280

[2] Mitrović, M., Popović, Đ.S., Naglić, D.T., Paro, J.N., Ilić, T. and Zavišić, B.K. (2014) Markers of Inflammation and Microvascular Complications in Type 1 Diabetes. Central European Journal of Medicine, 9, 748-753. http://dx.doi.org/10.2478/s11536-013-0335-6

[3] Ramachandran, A., Snehalatha, C., Shetty, A.S. and Nanditha, A. (2012) Trends in Prevalence of Diabetes in Asian Countries. World journal of Diabetes, 3, 110. http://dx.doi.org/10.4239/wjd.v3.i6.110

[4] Ferrante Do Amaral, C.E. and Wolf, B. (2008) Current Development in Non-Invasive Glucose Monitoring. Medical Engineering \& Physics, 30, 541-549. http://dx.doi.org/10.1016/j.medengphy.2007.06.003

[5] Vashist, S.K. (2012) Non-Invasive Glucose Monitoring Technology in Diabetes Management: A Review. Analytica Chimica Acta, 750, 16-27. http://dx.doi.org/10.1016/j.aca.2012.03.043

[6] Unnikrishna Menon, K.A., Hemachandran, D. and Abhishek, T.K. (2013) A Survey on Non-Invasive Blood Glucose Monitoring Using NIR. 2013 International Conference on Communications and Signal Processing (ICCSP), 10691072. http://dx.doi.org/10.1109/iccsp.2013.6577220

[7] Lam, S.C.H., Chung, J.W.Y., Fan, K.L. and Wong, T.K.S. (2010) Non-Invasive Blood Glucose Measurement by Near Infrared Spectroscopy: Machine Drift, Time Drift and Physiological Effect. Spectroscopy, 24. http://dx.doi.org/10.1155/2010/929506

[8] Maruo, K., Tsurugi, M., Chin, J., Ota, T., Arimoto, H., Yamada, Y., Tamura, M., Ishii, M. and Ozaki, Y. (2003) Noninvasive Blood Glucose Assay Using a Newly Developed Near-Infrared System. IEEE Journal of Selected Topics in Quantum Electronics, 9, 322-330. http://dx.doi.org/10.1109/JSTQE.2003.811283

[9] Malin, S.F., Ruchti, T.L., Blank, T.B., Thennadil, S.N. and Monfre, S.L. (1999) Noninvasive Prediction of Glucose by Near-Infrared Diffuse Reflectance Spectroscopy. Clinical Chemistry, 45, 1651-1658.

[10] Elgendi, M. (2012) On the Analysis of Fingertip Photoplethysmogram Signals. Current Cardiology Reviews, 8, 14. http://dx.doi.org/10.2174/157340312801215782

[11] Ubeyli, E.D., Cvetkovic, D. and Cosic, I. (2007) Eigenvector Methods for Analysis of Human PPG, ECG and EEG Signals. 29th Annual International Conference of the Engineering in Medicine and Biology Society, 3304-3307. http://dx.doi.org/10.1109/iembs.2007.4353036

[12] Du, Y.P., Liang, Y.Z., Kasemsumran, S., Maruo, K. and Ozaki, Y. (2004) Removal of Interference Signals Due to Water from in Vivo Near-Infrared (NIR) Spectra of Blood Glucose by Region Orthogonal Signal Correction (ROSC). Analytical Sciences, 20, 1339-1345. http://dx.doi.org/10.2116/analsci.20.1339

[13] Ramasahayam, S., Haindavi, K.S., Kavala, B. and Chowdhury, S.R. (2013) Non Invasive Estimation of Blood Glucose Using Near Infra Red Spectroscopy and Double Regression Analysis. 2013 Seventh International Conference on Sensing Technology (ICST), 627-631. http://dx.doi.org/10.1109/ICSensT.2013.6727729

[14] Yadav, J., Rani, A., Singh, V. and Murari, B.M. (2014) Near-Infrared LED Based Non-Invasive Blood Glucose Sensor. 2014 International Conference on Signal Processing and Integrated Networks (SPIN), 591-594. http://dx.doi.org/10.1109/SPIN.2014.6777023

[15] Barman, I., Kong, C.-R., Dingari, N.C., Dasari, R.R. and Feld, M.S. (2010) Development of Robust Calibration Models Using Support Vector Machines for Spectroscopic Monitoring of Blood Glucose. Analytical Chemistry, 82, 97199726. http://dx.doi.org/10.1021/ac101754n

[16] Ping, Z., Yingchun, L., Jie, M. and Siliang, M. (2005) Analysis of Noninvasive Measurement of Human Blood Glucose with ANN-NIR Spectroscopy. International Conference on Neural Networks and Brain, 1350-1353. 
http://dx.doi.org/10.1109/icnnb.2005.1614881

[17] Kovatchev, B.P., Gonder-Frederick, L.A., Cox, D.J. and Clarke, W.L. (2004) Evaluating the Accuracy of Continuous Glucose-Monitoring Sensors Continuous Glucose-Error Grid Analysis Illustrated by Therasense Freestyle Navigator Data. Diabetes Care, 27, 1922-1928. http://dx.doi.org/10.2337/diacare.27.8.1922 